\title{
Studien über die Wiederherstellung des Bluteiweisses sowie dessen kolloid=osmotischen Drucks im normalen und pathologischen Zustand.
}

\author{
V. Mitteilung: Versuche bei gestörter Schilddrüsenfunktion. \\ Von \\ Rijuro Fukuhara. \\ (福 原 利十郎) \\ (Aus der Medizinischen Klinik von Prof. Dr. T. Kato, \\ Tohoku-Reichsuniversität zu Sendai.)
}

\begin{abstract}
Dass die Schilddrüse, die eine wichtige Stellung im endokrinen System einnimmt, durch ihren hormonalen Einfluss den allgemeinen Stoffwechsel wesentlich steuert, kann man auch aus der Tatsache, dass bei der Base do w'schen Krankheit der Grundumsatz gesteigert wird, leicht ersehen. Schon von alters her ist die Tatsache, dass auch der Eiweissstoffwechsel durch Funktionsstörung der Schilddrüse mancherlei Veränderungen erleidet, allgemein bekannt und durch Untersuchungen von zahlreichen Forschern, wie Müller, Scholz, ${ }^{23}$ Magnus-Levy, ${ }^{3>}$ Beumer u. Iseki ${ }^{4)}$ Deusch ${ }^{5)}$ Frowein, ${ }^{6)}$ Abelin u. Sato, ${ }^{7)}$ Ueno, ${ }^{8)}$ Lichtwitz u. Conitzer, ${ }^{9}$ Ichikawa u. Sasaki, ${ }^{10)}$ Loeper, Lemaire, Lesure u. Tonnet, ${ }^{11}$ Láng, ${ }^{12)}$ Morioka ${ }^{13\rangle}$ u.a.m. erwiesen worden. Ferner auch an
\end{abstract}

\footnotetext{
1) Müller, Dtsch. Arch. f. klin. Med., 1893, 51, 335.

2) S cholz, Zentralbl. f. inn. Med., 1895, 1041.

3) M a g u s-Le v y, Ztschr. f. klin. Med., 1904, 52, 201.

4) Be umur u. Iseki, Berl. klin. Wschr, 1920, 178.

5) Deusch, Dtsch. Arch. f. klin. Med., 1920, 134, 342.

6) Frowei n, Ztschr. ges. exp. Med., 1921, 24, 162.

7) A belin u. Sato, Schweiz. med. Wschr., 1925, 45.

8) Ueno, Folia Endocrinol. Jap., 1926, 2, 805.

9) Lichtwitz u. Conitzer, Ztschr. ges. exp. Med., 1927, 56, 527.

10) Ichikawa u. S as aki, Folia Endocrinol. Jap., 1927-28, 3, 863.

11) Loeper, Lemaire, Lesure u. Tonnet, Progrés méd., 1930, 1965.

12) L áng, Ztschr. ges. exp. Med., 1933, 92, 102.

13) Morioka, Kyoto-Furitsu Ikadaigaku Zasshi, 1933, 7, 263.
} 
hiesiger Klinik haben Tada u. Nakazawa, ${ }^{14)}$ Hatafuku u. Naka$\mathrm{zawa}^{15)}$ Untersuchungen über die Beziehungen zwischen der Schilddrüsenfunktion und dem Eiweiss des Blutes sowie dessen kolloidosmotischen Druck angestellt und fanden, dass durch Injektionen von Thyreoglandol oder durch Fütterung von Schilddrüsenpräparat die Molekularaggregate der Bluteiweisskörper im allgemeinen verkleinert waren und dadurch der kolloid-osmotische Druck für 1\% Bluteiweiss erhöht war.

Die oben er'wähnte Beobachtung legt die Vermutung nahe, dass wenn am Tier, nachdem ihm die Funktion der Schilddrüse, die, wie oben angeführt, den Eiweisstoffwechsel wesentlich steuert, erhöht oder unterdrückt worden ist, die Plasmaphäresis ausgeführt wird, das Verhalten in der danach einsetzenden Wiederherstellung des Bluteiweisses und des k.o.D. irgendwie geändert werden müsste. Von diesem Gesichtspunkt ausgehend habe ich an Kaninchen, bei welchen der Hyperthyreoidismus durch Fütterung mit Schilddrüsenpräparat erzeugt oder der Athyreoidismus durch Schilddrüsenexstirpation herbeigeführt wurde, die Plasmaphäresis mit früher geschilderter $\mathrm{Me}$ thode ${ }^{16)}$ ausgeführt, und dann das Verhalten in der danach einsetzenden Wiederherstellung des Bluteiweisses und des k.o.D. untersucht.

Die Technik der Bestimmungen war dieselbe, wie in früherer Mitteilung ${ }^{16)}$ angegeben wurde.

1. Versuch an hyperthyreotischen Kaninchen.

Es wurden normalen gut ernährten Kaninchen von über $2 \mathrm{~kg}$ Körpergewicht je 0,2-0,4 g von Thyreoidinum siccum (Parke-Davis \& Co.) täglich einmal 3-4 Wochen lang verabreicht.

Um die Versuchsdauer nach der auszuführenden Plasmaphäresis hinlänglich protrahieren zu können, habe ich schon zu einer Zeit, wo hyperthyreotische Symptome, wenn auch schon in Erscheinung, doch noch nicht so manifest in den Vordergrund traten, je nach jeweiligem Zustand des betreffenden Tiers die Plasmaphäresis von $50-70 \mathrm{ccm}$ ausgeführt und dann das Verhalten in der Wiederherstellung des Bluteiweisses und des k. o. D. verfolgt. Die Fütterung mit Schilddrüsenpräparat wurde auch nach Ausführung der Plasmaphäresis noch

14) Tada u. Nakazawa, Tohoku Jonrn. Exp. Med., 1930, 15, 119.

15) Hatafuku u. Nakazawa, Ibid., 1933, 21, 107.

16) Fukuhara, Ibid., 1937, 30, 465. 
wie vor weiter fortgesetzt, aber das Tier ging meistens 72-120 Stunden nach der Plasmaphäresis ein.

In Tab. 1 sind an 5 Versuchen erhobene Resultate wiedergegeben.

In der Vorprobe konnten, wahrscheinlich wegen des noch nicht

\section{Tabelle 1.}

Eiweiss des Bluts und dessen kolloid-osmotischer Druck nach

Plasmaphäresis bei hyperthyreotischen Kaninchen.

\begin{tabular}{|c|c|c|c|c|c|c|c|c|c|c|c|}
\hline \multirow{2}{*}{ 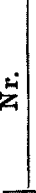 } & \multirow{2}{*}{$\begin{array}{l}\text { Zeit d. } \\
\text { Blut- } \\
\text { ent- } \\
\text { nahme } \\
\text { (Std.) }\end{array}$} & \multirow{2}{*}{$\begin{array}{l}\text { Körper- } \\
\text { gewicht } \\
\text { (g) u. } \\
\text { Gesch- } \\
\text { lecht }\end{array}$} & \multicolumn{2}{|c|}{$\begin{array}{l}\text { Hämo- } \\
\text { globin }\end{array}$} & \multicolumn{2}{|c|}{$\begin{array}{l}\text { Serum- } \\
\text { eiweiss }\end{array}$} & \multicolumn{2}{|c|}{$\begin{array}{l}\text { Kolloid-os- } \\
\text { mot. Druck }\end{array}$} & \multicolumn{2}{|c|}{$\begin{array}{c}\text { Kolloid- } \\
\text { osmot. } \\
\text { Druck pro\% }\end{array}$} & \multirow{2}{*}{ Bemerkungen } \\
\hline & & & $(g / d l)$ & $\left|\begin{array}{l}\text { Diff. } \\
\text { in } \%\end{array}\right|$ & $(\%)$ & $\begin{array}{l}\text { Diff. } \\
\text { in } \%\end{array}$ & $\left(\begin{array}{l}\mathrm{mm} \\
\mathrm{H}_{2} \mathrm{O}\end{array}\right)$ & $\begin{array}{l}\text { Diff. } \\
\text { in } \%\end{array}$ & $\left(\begin{array}{l}\mathrm{mm} \\
\mathrm{H}_{2} \mathrm{O}\end{array}\right)$ & $\left|\begin{array}{c}\text { Diff. } \\
\text { in } \%\end{array}\right|$ & \\
\hline & Plast & $1630 ٪$ & 16,48 & & 5,51 & & 239 & & 43,4 & & 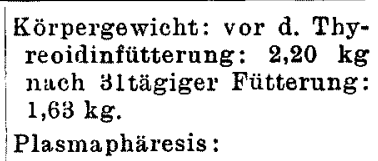 \\
\hline 1 & $\begin{array}{cc}\text { Nach } & 3 \\
" & 6 \\
" & 24 \\
" & 30 \\
" & 48 \\
" & 72 \\
" & 96\end{array}$ & $\begin{array}{l}1590 \\
1660 \\
1620 \\
1500\end{array}$ & $\left|\begin{array}{l}16,78 \\
16,48 \\
15,92 \\
15,92 \\
15,36 \\
15,36 \\
15,36\end{array}\right|$ & $\begin{array}{l}+1,8 \\
\pm 0 \\
-3,4 \\
-3,4 \\
-6,8 \\
-6,8 \\
-6,8\end{array}$ & $\begin{array}{l}3,98 \\
3,92 \\
5,10 \\
5,29 \\
5,94 \\
5,97 \\
6,30\end{array}$ & $\begin{array}{r}-27,8 \\
-28,8 \\
-7,4 \\
-\quad 4,0 \\
+7,8 \\
+\quad 8,3 \\
+14,3\end{array}$ & $\begin{array}{l}165 \\
169 \\
199 \\
191 \\
213 \\
217 \\
214\end{array}$ & $\begin{array}{l}-31,0 \\
-29,3 \\
-16,7 \\
-20,1 \\
-10,9 \\
-\quad 9,2 \\
-10,5\end{array}$ & $\begin{array}{l}41,4 \\
43,1 \\
39,0 \\
36,1 \\
35,8 \\
36,3 \\
34,0\end{array}$ & $\begin{array}{r}4,6 \\
-0,7 \\
-10,1 \\
-16,8 \\
-17,5 \\
-16,3 \\
-21,6\end{array}$ & $\begin{array}{l}\text { Starb } 96 \text { Std. nach Plasma- } \\
\text { phäresis. }\end{array}$ \\
\hline & Plasr & $1950 \delta$ & 14,80 & & 6,03 & & 258 & & 42,8 & & $\begin{array}{l}\text { Körpergewicht: vor d. Thy- } \\
\text { reoidinfütterung: } 2,38 \mathrm{~kg} \\
\text { nach } 22 \text { tägiger Fütterung: } \\
1,95 \mathrm{~kg} \text {. } \\
\text { Plasmaphäresis: }\end{array}$ \\
\hline 2 & $\begin{array}{cr}\text { Nach } & 3 \\
" & 6 \\
" & 24 \\
" & 30 \\
" & 48 \\
" & 72 \\
" & 96\end{array}$ & $\begin{array}{l}1820 \\
1680 \\
1420\end{array}$ & $\left|\begin{array}{l}14,80 \\
14,80 \\
14,52 \\
14,52 \\
13,94 \\
14,24 \\
13,94\end{array}\right|$ & $\begin{array}{l} \pm 0 \\
\pm 0 \\
-1,9 \\
-1,9 \\
-5,8 \\
-3,8 \\
-5,8\end{array}$ & $\begin{array}{l}4,12 \\
4,05 \\
5,43 \\
5,49 \\
6,08 \\
5,97 \\
6,14\end{array}$ & $\begin{array}{r}-31,7 \\
-32,8 \\
-\quad 9,9 \\
-8,9 \\
+\quad 0,8 \\
-\quad 1,0 \\
+\quad 1,8\end{array}$ & $\begin{array}{l}161 \\
162 \\
206 \\
200 \\
199 \\
208 \\
188\end{array}$ & $\begin{array}{l}-37,6 \\
-37,2 \\
-20,1 \\
-22,5 \\
-22,9 \\
-19,4 \\
-27,1\end{array}$ & $\begin{array}{l}39,1 \\
40,0 \\
37,9 \\
36,4 \\
32,7 \\
34,8 \\
30,6\end{array}$ & $\begin{array}{r}-8,6 \\
-6,5 \\
-11,4 \\
-14,9 \\
-23,6 \\
-18,7 \\
-28,5\end{array}$ & $\begin{array}{l}\text { Starb } 98 \text { Std. nach Plasma- } \\
\text { phäresis. }\end{array}$ \\
\hline & Plasi & $1570 \hat{~}$ & $\mid 17,36$ & & $|5,60|$ & & 248 & & 44,3 & & $\begin{array}{l}\text { Körpetgewicht: vor d. Thy- } \\
\text { reoidinfütterung: } \mathbf{2 , 2 7} \mathbf{~ k g} \\
\text { nach 20tägiger Futterung: } \\
1,57 \mathrm{~kg} \text {. } \\
\text { Plasmaphäresis: }\end{array}$ \\
\hline 3 & $\begin{array}{cr}\text { Nach } & 3 \\
" & 6 \\
" & 24 \\
" & 30 \\
" & 48 \\
" & 72 \\
" & 96 \\
" & 120\end{array}$ & $\begin{array}{l}1460 \\
1450 \\
1440 \\
1320\end{array}$ & $\left|\begin{array}{l}17,36 \\
17,36 \\
16,78 \\
16,78 \\
16,20 \\
15,92 \\
15,64 \\
15,64\end{array}\right|$ & $\begin{array}{l} \pm 0 \\
\pm 0 \\
-3,3 \\
-3,3 \\
-6,7 \\
-8,3 \\
-9,9 \\
-9,9\end{array}$ & $\begin{array}{l}4,34 \\
4,40 \\
5,29 \\
5,36 \\
5,53 \\
5,64 \\
5,81 \\
5,67\end{array}$ & $\begin{array}{r}-22,5 \\
-21,4 \\
-\quad 5,5 \\
-\quad 4,3 \\
-\quad 1,2 \\
+\quad 0,7 \\
+\quad 3,7 \\
+\quad 1,2\end{array}$ & \begin{tabular}{l|}
174 \\
175 \\
199 \\
192 \\
199 \\
193 \\
193 \\
191
\end{tabular} & $\begin{array}{l}-29,8 \\
-29,4 \\
-19,7 \\
-22,6 \\
-19,7 \\
-22,2 \\
-22,2 \\
-23,0\end{array}$ & $\begin{array}{l}40,1 \\
39,8 \\
37,6 \\
35,8 \\
36,0 \\
34,2 \\
33,2 \\
33,7\end{array}$ & $\begin{array}{r}9,5 \\
-10,1 \\
-15,1 \\
-19,2 \\
-18,7 \\
-22,8 \\
-25,0 \\
-23,9\end{array}$ & Starb 120 Std. nach Plasma- \\
\hline
\end{tabular}




\begin{tabular}{|c|c|c|c|c|c|c|c|c|c|c|c|}
\hline \multirow{2}{*}{$\dot{\mathrm{z}}$} & \multirow{2}{*}{$\begin{array}{l}\text { Zeit d. } \\
\text { Blut- } \\
\text { ent- } \\
\text { nahme } \\
\text { (Std.) }\end{array}$} & \multirow{2}{*}{$\begin{array}{l}\text { Körper- } \\
\text { gewicht } \\
\text { (g) u. } \\
\text { Gesch- } \\
\text { lecht }\end{array}$} & \multicolumn{2}{|c|}{$\begin{array}{l}\text { Hämo- } \\
\text { globin }\end{array}$} & \multicolumn{2}{|c|}{$\begin{array}{l}\text { Serum- } \\
\text { eiweiss }\end{array}$} & \multicolumn{2}{|c|}{$\begin{array}{l}\text { Kolloid-os- } \\
\text { mot. Druck }\end{array}$} & \multicolumn{2}{|c|}{$\mid \begin{array}{c}\text { Kolloid- } \\
\text { osmot. } \\
\text { Druck pro } \%\end{array}$} & \multirow{2}{*}{ Bemerkungen } \\
\hline & & & $(\mathrm{g} / \mathrm{dr})$ & $\begin{array}{l}\text { Diff: } \\
\text { in } \%\end{array}$ & $(\%)$ & $\begin{array}{l}\text { Diff. } \\
\text { in } \%\end{array}$ & $\left(\begin{array}{l}\mathrm{mm}_{\mathrm{H}_{2} \mathrm{O}} \\
)\end{array}\right)$ & $\begin{array}{l}\text { Diff. } \\
\text { in } \%\end{array}$ & $\left|\left(\mathrm{~mm}_{2} \mathrm{O}\right)\right|$ & $\begin{array}{l}\text { Diff. } \\
\text { in } \%\end{array}$ & \\
\hline & Plasm & $1330 \mathrm{f}$ & $|17,08|$ & & 5,58 & & 244 & & 43,7 & & $\begin{array}{l}\text { Körpergewicht: vor d. Thy- } \\
\text { reoidinfütterung: 2,15 kg } \\
\text { nach 28tägiger Fütterung: } \\
1,33 \mathrm{~kg} \text {. }\end{array}$ \\
\hline 4 & $\begin{array}{cr}\text { Nach } & 3 \\
" & 6 \\
" & 24 \\
" & 30 \\
" & 48 \\
" & 72\end{array}$ & $\begin{array}{l}1300 \\
1250 \\
1180\end{array}$ & $\begin{array}{l}17,36 \\
17,36 \\
16,78 \\
16,78 \\
16,20 \\
15,64\end{array}$ & $\begin{array}{r}+1,6 \\
+1,6 \\
-1,8 \\
-1,8 \\
-5,1 \\
-8,4\end{array}$ & $\begin{array}{l}3,63 \\
3,59 \\
5,32 \\
5,27 \\
5,66 \\
5,70\end{array}$ & $\begin{array}{r}-34,9 \\
-35,7 \\
-\quad 4,6 \\
-5,5 \\
+\quad 1,4 \\
+\quad 2,1\end{array}$ & $\begin{array}{l}143 \\
140 \\
189 \\
187 \\
178 \\
184\end{array}$ & $\begin{array}{l}-41,4 \\
-42,6 \\
-22,5 \\
-23,4 \\
-27,0 \\
-24,6\end{array}$ & $\begin{array}{l}39,4 \\
39,0 \\
35,5 \\
35,5 \\
31,4 \\
32,3\end{array}$ & $\begin{array}{l}-9,8 \\
-10,7 \\
-18,8 \\
-18,8 \\
-28,1 \\
-26,1\end{array}$ & $\begin{array}{l}\text { Starb } 75 \text { Std. nach Plasma- } \\
\text { phäresis. }\end{array}$ \\
\hline & Plasm & $1300 \precsim$ & 15,64 & & 5,99 & & 246 & & 41,0 & & $\begin{array}{l}\text { Körpergewicht: vor d. Thळ- } \\
\text { reoidinfütterung: } 2,23 \mathrm{~kg} \\
\text { nach 26tägiger Fütterng } \\
1,30 \mathrm{~kg} \text {. } \\
\text { Plasmaphäresis: }\end{array}$ \\
\hline 5 & $\begin{array}{cr}\text { Nach } & 3 \\
" & 6 \\
" & 24 \\
" & 30 \\
" & 48 \\
" & 72\end{array}$ & $\begin{array}{l}1430 \\
1280 \\
1220\end{array}$ & $\begin{array}{l}15,64 \\
15,36 \\
15,08 \\
15,08 \\
15,08 \\
14,80\end{array}$ & $\begin{array}{l} \pm 0 \\
-1,8 \\
-3,6 \\
-3,6 \\
-3,6 \\
-5,4\end{array}$ & $\begin{array}{l}4,96 \\
4,88 \\
5,36 \\
5,64 \\
5,74 \\
6,08 \\
\end{array}$ & $\begin{array}{r}-17,2 \\
-18,5 \\
-10,5 \\
-\quad 7,5 \\
-4,2 \\
+\quad 1,5\end{array}$ & $\begin{array}{l}186 \\
182 \\
191 \\
198 \\
189 \\
191\end{array}$ & $\begin{array}{l}-24,4 \\
-26,0 \\
-22,3 \\
-19,5 \\
-23,2 \\
-22,3\end{array}$ & $\begin{array}{l}37,5 \\
37,3 \\
35,6 \\
35,1 \\
32,9 \\
31,4\end{array}$ & $\begin{array}{r}-8,5 \\
-9,0 \\
-13,2 \\
-14,4 \\
-19,7 \\
-23,4\end{array}$ & $\begin{array}{l}\text { Blutentnahme } 50 \mathrm{ccm} \text {. } \\
\text { Blutreinjektion } 50 \mathrm{ccm} \text {. }\end{array}$ \\
\hline
\end{tabular}

so vorgeschrittenen Hyperthyreoidismus, keine merklichen Veränderungen des Bluteiweisses und des k.o.D. nachgewiesen werden. 3 Std. nach Ausfülırung der Plasmaphäresis betrug die Abnahme des Bluteiweisses $17,2-34,9 \%$, dieselbe des k. o. D. 24,4-41,4\%, beide erfuhren also gemeinschaftlich erhebliche Abnahme. Da aber die Senkung des letzteren etwas erheblicher war, zeigte der Druck pro \% eine Erniedrigung von 4,6-9,8\%.

In 6stündigem Ablauf blieb beides im grossen ganzen auf derselben Höhe stehen; nach 24-30 Std. befand sich das Eiweiss augenfällig auf dem Weg zur Wiederherstellung, kam dennoch meistens nicht bis zum Anfangswert zurück. Die Wiederherstellung des k.o.D. war beträchtlich protrahiert, weshalb der Druck pro \% weiter auffallend absinkend, endlich sich um eine Abnahme von ungefähr 15\% herum bewegte.

U̇berblicken wir den Verlauf der nach 30 Std. auftretenden Veränderungen in Kürze, so lässt sich folgendes sagen: Das Bluteiweiss tritt unter allmählicher Annäherung an den Anfangswert nach $48 \mathrm{Std}$. 
bei nahe an den Ursprungswert heran, und bleibt daselbst stehen, ohne meistens denselben überschritten zu haben. Was die Wiederherstellung des k.o.D. anbelangt, weist er nach 30 Std. entweder fast keine Tendenz zur Wiederherstellung oder die Neigung zu äusserst spärlicher, transitorischer Rückkehr auf, mithin erniedrigt sich der Druck pro \% ausserordentlich, und so bis zum nach 72-120 Std. eingetretenen Tod beharren der k.o.D. und Druck pro \% gemeinschaftlich auf erniedrigten Werten. Hämoglobin nimmt gleichzeitig mit Blutentnahme mehr oder minder $a b$, aber in höchst bescheidenem Masse. Fig. 1 veranschaulicht den an Versuch 2 beobachteten Verlauf als Beispiel hierfür in Kurven.

Aus obigen Auseinandersetzungen geht also hervor, dass beim hyperthyreotischen Kaninchen das Bluteiweiss zwar in quantitativer Hinsicht, aber nicht in

Fig. 1. Prozentnelle Verïnderungen des Hämoglobins, des Serumei weisses, des kolloidosmotischen Drucks und des Drucks pro \% des Bluts nach Plasmaphäresis bei einem lyyperthyreotischen Kaninchen (Versuch 2).

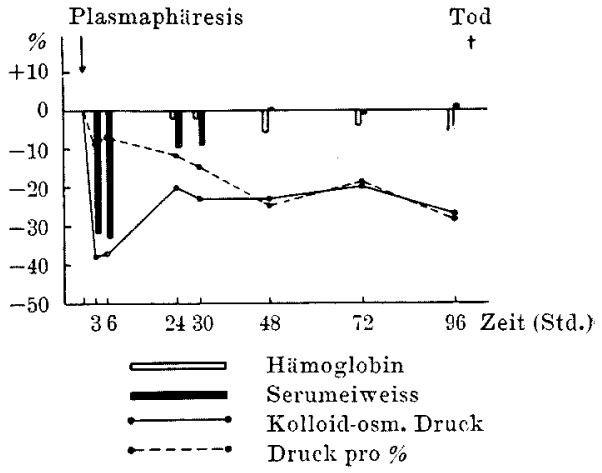
qualitativer Hinsicht wiederhergestellt werden kann, weil hierbei reife, osmoaktive kleine Eiweissteilchen nicht genügend zur Verfügung stehen, wobei k.o.D. und Druck pro \% naturgemäss erniedrigte Werte beibehalten.

2. Versuch an athyreotischen Kaninchen.

An ungefähr $2 \mathrm{~kg}$ schweren Kaninchen, denen die Schilddrüse total exstirpiert war und welche nach ca. 3-4 Wochen das thyreoprive Syndrom darboten, habe ich die Plasmaphäresis von $50-70 \mathrm{ccm}$ ausgeführt und das Verhalten in der danach auftretenden Wiederherstellung des Bluteiweisses und des k. o. D. beobachtet. In Tab. 2 sind an 5 Kaninchen erhobene Ergebnisse zusammengestellt.

In der Vorprobe nahm Hämoglobin etwas ab, der Gehalt an Bluteiweiss war anscheinend mehr oder weniger höher als in der Norm, während der k.o.D. keine bemerkbare Veränderung aufwies. 3 Std. nach Ausführung der Plasmaphäresis erfuhr das Bluteiweiss eine Ab- 


\section{Tabelle 2.}

Eiweiss des Bluts und dessen kolloid-osmotische Druck nach Plasmaphäresis bei athyreotischen Kaninchen.

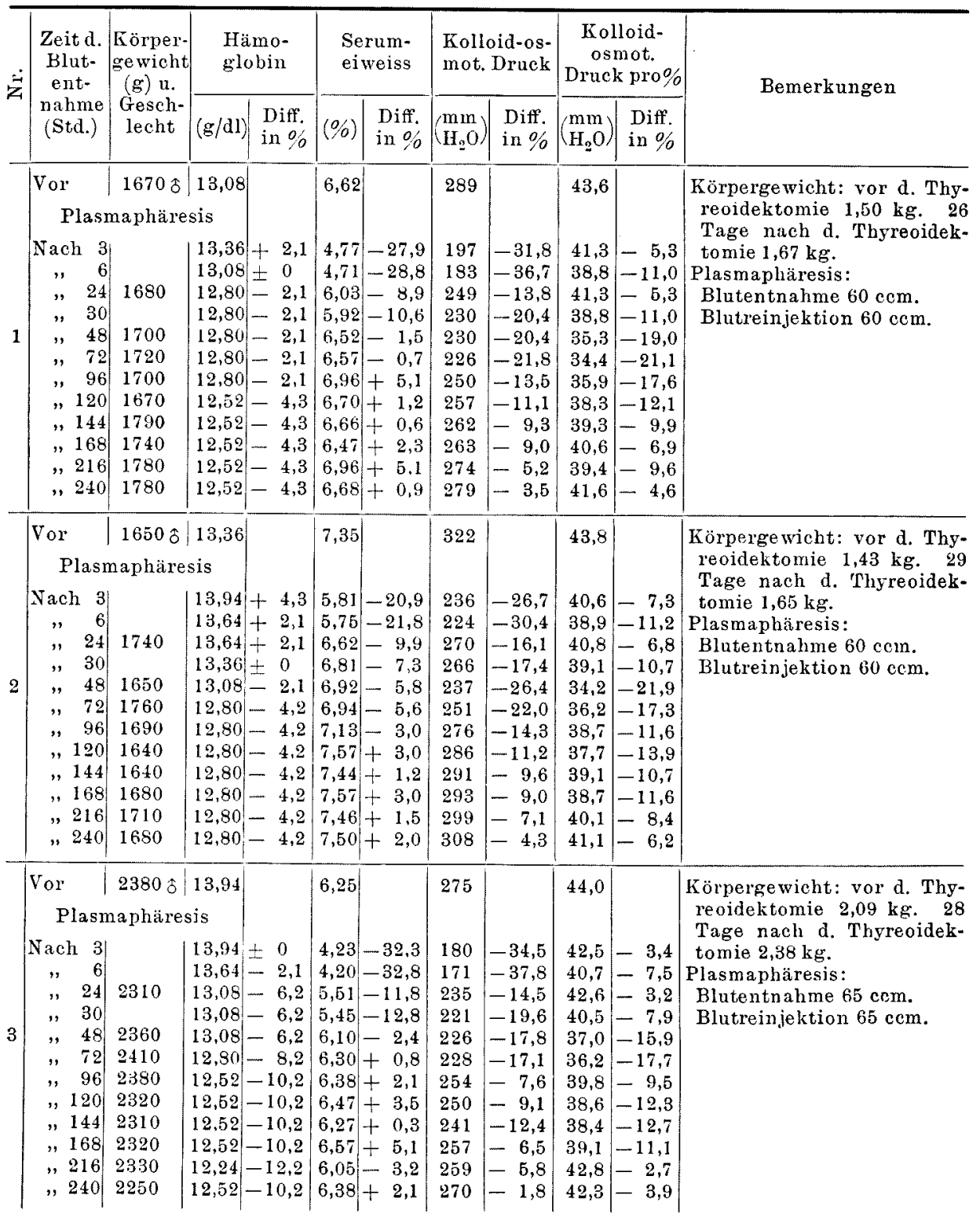




\begin{tabular}{|c|c|c|c|c|c|c|c|c|c|c|c|}
\hline \multirow[t]{2}{*}{$\dot{8}$} & \multirow{2}{*}{$\begin{array}{c}\text { Zeit d. } \\
\text { Blut- } \\
\text { ent- } \\
\text { nahme } \\
\text { (Std.) }\end{array}$} & \multirow{2}{*}{$\begin{array}{c}\text { Körper- } \\
\text { gewicht } \\
\text { (g) u. } \\
\text { Geseh- } \\
\text { lecht }\end{array}$} & \multicolumn{2}{|c|}{$\begin{array}{l}\text { Hämo- } \\
\text { globin }\end{array}$} & \multicolumn{2}{|c|}{$\begin{array}{l}\text { Serum- } \\
\text { eiweiss }\end{array}$} & \multicolumn{2}{|c|}{$\begin{array}{l}\text { Kolloid-os- } \\
\text { mot. Druck }\end{array}$} & \multicolumn{2}{|c|}{$\begin{array}{c}\text { Kolloid- } \\
\text { osmot. } \\
\text { Druck pio\% }\end{array}$} & \multirow{2}{*}{ Bemerkungen } \\
\hline & & & $(g / d)$ & $\begin{array}{l}\text { Diff. } \\
\text { in } \%\end{array}$ & $(\%)$ & $\begin{array}{l}\text { Diff. } \\
\text { in } \%\end{array}$ & $\left(\begin{array}{l}\mathrm{mm} \\
\mathrm{H}_{2} \mathrm{O}\end{array}\right)$ & $\begin{array}{l}\text { Diff. } \\
\text { in } \%\end{array}$ & $\left(\begin{array}{l}\mathrm{mm}_{\mathrm{H}_{2} \mathrm{O}} \\
)\end{array}\right)$ & $\begin{array}{l}\text { Diff. } \\
\text { in } \%\end{array}$ & \\
\hline \multirow[b]{2}{*}{4} & $\begin{array}{l}\text { Vor } \\
\text { Plasm }\end{array}$ & $\begin{array}{l}2270 \delta \\
\text { maphäres }\end{array}$ & $\begin{array}{l}14,24 \\
\text { sis }\end{array}$ & & 6,08 & & 263 & & 43,2 & & \multirow{2}{*}{$\begin{array}{l}\text { Körpergewicht: vor d. Thy- } \\
\text { reoidektomie } 1,92 \mathrm{~kg} \text {. } 25 \\
\text { Tage nach d. Thyreoidek- } \\
\text { tomie } 2,27 \mathrm{~kg} \text {. } \\
\text { Plasmaphäresis: } \\
\text { Blutentrahme } 70 \mathrm{ccm} \text {. } \\
\text { Blutreinjektion } 70 \mathrm{ccm} \text {. }\end{array}$} \\
\hline & $\mid \begin{array}{cr}\text { Nach } & 3 \\
" & 6 \\
" & 24 \\
" & 30 \\
" & 48 \\
" & 72 \\
" & 96 \\
" 120 & 120 \\
" 1 & 144 \\
" 168 \\
" 1216 \\
" 240\end{array}$ & $\begin{array}{l}2180 \\
2240 \\
2230 \\
2210 \\
2300 \\
2230 \\
2270 \\
2250\end{array}$ & $\begin{array}{l}13,64 \\
13,64 \\
13,08 \\
13,08 \\
12,80 \\
12,52 \\
12,24 \\
12,24 \\
12,24 \\
12,24 \\
12,52 \\
12,24\end{array}$ & $\begin{array}{l}-4,2 \\
-\quad 4,2 \\
-8,1 \\
-8,1 \\
-10,1 \\
-12,1 \\
-14,0 \\
-14,0 \\
-14,0 \\
-14,0 \\
-12,1 \\
-14,0\end{array}$ & $\begin{array}{l}3,76 \\
3,81 \\
4,99 \\
5,16 \\
5,75 \\
6,10 \\
6,57 \\
6,20 \\
6,27 \\
5,88 \\
5,99 \\
6,25\end{array} \mid$ & $\begin{array}{r}-38,1 \\
-37,3 \\
-17,9 \\
-15,1 \\
-\quad 5,4 \\
+\quad 0,3 \\
+\quad 8,0 \\
+\quad 2,0 \\
+\quad 3,1 \\
-\quad 3,3 \\
-\quad 1,5 \\
+\quad 2,8\end{array}$ & $\begin{array}{l}150 \\
151 \\
197 \\
200 \\
214 \\
221 \\
245 \\
243 \\
249 \\
247 \\
247 \\
252\end{array}$ & $\begin{array}{r}-43,0 \\
-42,6 \\
-25,1 \\
-23,9 \\
-18,6 \\
-16,0 \\
-6,8 \\
-7,6 \\
-\quad 5,3 \\
-6,1 \\
-6,1 \\
-4,2\end{array}$ & $\begin{array}{l}39,9 \\
39,6 \\
39,5 \\
38,7 \\
37,2 \\
36,2 \\
37,3 \\
39,9 \\
39,7 \\
42,0 \\
41,2 \\
40,3\end{array}$ & $\begin{array}{r}7,6 \\
-8,3 \\
-8,6 \\
-10,4 \\
-13,9 \\
-16,2 \\
-13,7 \\
-9,9 \\
-8,1 \\
-\quad 2,8 \\
-4,6 \\
-6,7\end{array}$ & \\
\hline \multirow[b]{2}{*}{5} & \begin{tabular}{|} 
Vor \\
Plasm
\end{tabular} & $\begin{array}{l}\mid 2220 \text { ठ } \\
\text { maphäre }\end{array}$ & 13,94 & & 5,88 & & 255 & & 43,4 & & $\begin{array}{l}\text { Körpergewicht: vor d. Thy- } \\
\text { reoidektomie } 1,90 \mathrm{~kg} \text {. } \mathbf{9 4} \\
\text { Tage nach d. Thyreoidek- }\end{array}$ \\
\hline & $\mid \begin{array}{cr}\text { Nach } & 3 \\
" & 6 \\
" & 24 \\
" & 30 \\
" & 48 \\
" & 72 \\
" & 96 \\
" 1 & 120 \\
" 144 \\
" 168 \\
" 1216 \\
" 240\end{array}$ & $\begin{array}{l}2130 \\
2190 \\
2030 \\
2110 \\
2130 \\
2020 \\
2010 \\
2000 \\
2110\end{array}$ & $\begin{array}{l}14,24 \\
13,64 \\
13,64 \\
13,36 \\
13,36 \\
13,08 \\
12,80 \\
12,80 \\
12,52 \\
12,52 \\
12,24 \\
12,24\end{array}$ & $\begin{array}{rr}+ & 2,1 \\
- & 2,1 \\
- & 2,1 \\
- & 4,2 \\
- & 4,2 \\
- & 6,2 \\
- & 8,2 \\
- & 8,2 \\
-10,2 \\
-10,2 \\
-12,2 \\
-12,2\end{array}$ & $\begin{array}{l}4,83 \\
4,64 \\
5,64 \\
5,58 \\
6,27 \\
6,16 \\
6,16 \\
6,45 \\
5,94 \\
6,03 \\
5,88 \\
5,72\end{array}$ & $\begin{array}{l}-17,8 \\
-21,1 \\
-\quad 4,1 \\
-5,1 \\
+\quad 6,6 \\
+\quad 4,8 \\
+\quad 4,8 \\
+10,0 \\
+1,0 \\
+2,5 \\
\pm 0 \\
-2,7\end{array}$ & $\begin{array}{l}191 \\
181 \\
240 \\
233 \\
240 \\
230 \\
238 \\
234 \\
230 \\
234 \\
237 \\
236\end{array}$ & $\begin{array}{r}-25,1 \\
-29,0 \\
-5,9 \\
-\quad 8,6 \\
-\quad 5,9 \\
-9,8 \\
-6,7 \\
-8,2 \\
-9,8 \\
-8,2 \\
-7,0 \\
-7,4\end{array}$ & $\begin{array}{l}39,5 \\
39,0 \\
42,5 \\
41,7 \\
38,3 \\
37,3 \\
38,6 \\
36,3 \\
38,7 \\
38,8 \\
40,3 \\
41,2\end{array}$ & $\begin{array}{r}-9,0 \\
-10,1 \\
-2,1 \\
-3,9 \\
-11,7 \\
-14,0 \\
-11,0 \\
-16,3 \\
-10,8 \\
-10,6 \\
-7,1 \\
-5,1\end{array}$ & $\begin{array}{l}\text { tomio } 2,22 \mathrm{~kg} . \\
\text { Plasmapharesis: } \\
\text { Blutentnahme } 60 \mathrm{ccm} . \\
\text { Blutreinjektion } 60 \mathrm{ccm} .\end{array}$ \\
\hline \multirow[b]{2}{*}{6} & $\begin{array}{l}\text { Vor } \\
\text { Plas }\end{array}$ & \begin{tabular}{|}
2380 甲 \\
maphäres
\end{tabular} & $\begin{array}{l}14,24 \\
\text { sis }\end{array}$ & & 6,47 & & 294 & & 45,4 & & \multirow{2}{*}{$\begin{array}{l}\text { Körpergewicht: vor d. Thy- } \\
\text { reoidektomie } 2,12 \mathrm{~kg} \text {. } 26 \\
\text { Tage nach d. Thyreoidek- } \\
\text { tomie } 2.38 \mathrm{~kg} \text {. } \\
\text { Plasmaphäresis : } \\
\text { Blutentnahme } 60 \mathrm{~cm} \text {. } \\
\text { Blutreinjektion } 60 \mathrm{cem} \text {. }\end{array}$} \\
\hline & $\mid \begin{array}{cr}\text { Nach } & 3 \\
" & 6 \\
" & 24 \\
\Rightarrow & 30 \\
" & 48 \\
" & 72 \\
" & 96 \\
" 120 \\
" 144 \\
" 168 \\
" 216 \\
" 2240\end{array}$ & $\begin{array}{l}2240 \\
2230 \\
2100 \\
1990 \\
1930 \\
2020 \\
2200 \\
2210\end{array}$ & $\begin{array}{l}13,94 \\
13,94 \\
13,36 \\
13,36 \\
13,08 \\
12,80 \\
12,80 \\
12,24 \\
12,24 \\
12,24 \\
12,24 \\
12,52\end{array}$ & $\begin{array}{l}-2,1 \\
-2,1 \\
-6,2 \\
-6,2 \\
-8,1 \\
-10,1 \\
-10,1 \\
-14,0 \\
-14,0 \\
-14,0 \\
-14,0 \\
-12,1\end{array}$ & $\begin{array}{l}4,77 \\
4,71 \\
6,23 \\
6,08 \\
6,49 \\
6,68 \\
6,19 \\
5,94 \\
5,97 \\
5,88 \\
6,05 \\
6,30\end{array}$ & $\begin{array}{r}-26,3 \\
-27,2 \\
-3,7 \\
-6,0 \\
+0,3 \\
+3,2 \\
-4,3 \\
-8,2 \\
-7,7 \\
-9,1 \\
-6,5 \\
-2,6\end{array}$ & $\begin{array}{l}209 \\
197 \\
262 \\
253 \\
248 \\
249 \\
247 \\
241 \\
237 \\
242 \\
251 \\
261\end{array}$ & $\begin{array}{l}-28,9 \\
-33,0 \\
-10,9 \\
-13,9 \\
-15,6 \\
-15,3 \\
-16,0 \\
-18,0 \\
-19,4 \\
-17,7 \\
-14,6 \\
-11,2\end{array}$ & $\begin{array}{l}43,8 \\
41,8 \\
42,0 \\
41,6 \\
38,2 \\
37,3 \\
39,9 \\
40,6 \\
39,7 \\
41,1 \\
41,5 \\
41,4\end{array}$ & $\mid \begin{array}{r}-3,5 \\
-7,9 \\
-7,5 \\
-8,4 \\
-15,8 \\
-17,8 \\
-12,1 \\
-10,6 \\
-12,5 \\
-9,5 \\
-8,6 \\
-8,8\end{array}$ & \\
\hline
\end{tabular}

nahme von $17,8-38,1 \%$, der k. o.D. von $25,1-43,0 \%$. Da die prozentuelle Abnahme des k. o. D. stärker als die des Bluteiweisses erfolgte, wies der Druck pro \% einen niedrigen Wert von $3,4-9,0 \%$ auf. 
Nach 24-30 Std. hatten das Bluteiweiss und der k. o. D. die Tendenz zu etlicher Wiederherstellung, der Druck pro \% erhöhte sich mitunter in geringem Masse.

Der Verlauf der nachträglichen Veränderungen lässt sich wie folgt zusammenfassen: Die Wiederherstellung des Bluteiweisses findet im allgemeinen gegenüber der Norm beträchtlich verzögert statt, indem es nämlich frühestens nach 48-72 Std. spätestenfalls erst in annähernd 100stündigem Ablauf den Ursprungswert erreicht und sich dann um denselben herum bewegt. Demgegenüber rückt der k. o. D. nach 24-30 Std. im allgemeinen ungemein träge dem Anfangswert näher, oder nachdem er sich nach 24 Std. vorübergehend noch

Fir. 2. Prozentuelle Veränderungen des Hämoglobins, des Serumeiweisses, des kolloid-osmotischen Drucks und des Drucks pro \% des Bluts nach Pläsmaphäresis bei einem athyreotischen Kaninchen (Versuch 1 ).

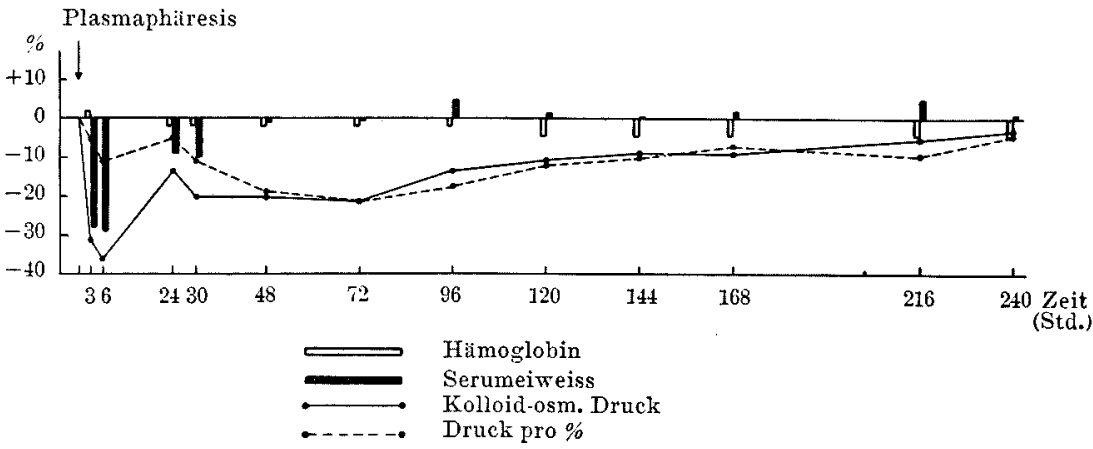

weiter erniedrigt hatte, schlägt er von 100 stündigem Ablauf an den Weg zur Wiederherstellung ein, ohne dennoch bis auf das frühere Niveau zurückzukommen. Mithin zieht sich die Wiederherstellung des Drucks pro \% ausserordentlich in die Länge. 10 Tage nach Ausführung der Plasmaphäresis treten Eiweiss, k. o. D. und Druck pro \% ebenfalls an die Anfangswerte heran, ohne dass sich jedoch k. o.D. und Druck pro \% völlig wiederherstellen können. Die Abnahme des Hämoglobins ist geringfügig, was wahrscheinlich auf geringgradige Anämie durch Blutentnahme zurückzuführen ist.

Fig. 2 bringt den Verlauf der an Versuch 1 beobachteten Veränderungen als Beispiel in Kurven zur Anschauung.

Beim athyreotischen Kaninchen ist also die Wiederherstellung des Bluteiweisses und des k.o.D. nach Ausführung der Plasmaphäresis beträchtlich verzögert, im besonderen aber die Wiederherstellung 
des k. o. D. ist derart zauderhaft, dass er selbst 10 Tage nach Ausführung der Plasmaphäresis noch nicht vollkommen den Anfangswert erreicht. Dementsprechend ist die Wiederherstellung des Drucks pro $\%$ überaus verzögert.

\section{Zusammenfassung und Besprechung.}

Bei der Übersicht über das Verhalten in der Wiederherstellung des Bluteiweisses und des k.o.D., welche am hyperthyreotischen Kaninchen nach Ausführung der Plasmaphäresis zustande kommt, fällt folgendes auf: das Bluteiweiss kommt im grossen ganzen ungefähr nach 48 Stunden bis zum Anfangswert zurück und bleibt von diesem Zeitpunkt an bis zum Eintritt des Todes, olne erhebliche Schwankungen, daselbst stehen. Im Gegensatz hierzu zeigt der k. o. D. des Blutes, wenn er sich auch bis zum 24stündigen Ablauf im Verein mit Zunahme des Eiweisses mehr oder weniger erhebt, dennoch beträchtlich niedrige Werte. Später erhöht er sich entweder nicht mehr, oder sinkt eher allmählich ab. Hand in Hand damit fällt auch der Druck pro $\%$ stufenweise herab.

Die entsprechende Beobachtung an athyreotischen Kaninchen ergibt sich folgendermassen: In diesem Fall ist die Wiederherstellung des Bluteiweisses im Vergleich mit derselben beim $\mathrm{H}_{y}$ perthyreoidismus noch mehr verzögert, indem es meistens erst nach 72 Std. den Ursprungswert erreicht und sich danach um denselben herum bewegt. K.o. D. ist bis zu 24stündigem Ablauf zu relativ prompter Erholung geneigt, und doch dem Ursprungswert unterlegen, um dann vorübergehend weiter noch abzusinken und mit ganz trägem Schritt in die Höhe zu steigen. Nach der Beobachtung, welche bis zu zehntägigem Ablauf nach Ausführung der Plasmaphäresis währte, beurteilt, pflegen der k.o.D. und Druck pro\% Anfangswerte noch nicht zu erreichen, ob das Bluteiweiss schon zur selben Zeit bis zu demselben zurückkommt.

Es erhebt sich dann die Frage, warum bei derart gestörter Schilddrüsenfunktion das Verhalten in der Wiederherstellung des Bluteiweisses, des k.o.D. und des Drucks pro \% nach Ausführung. der Plasmaphäresis von der Norm abweicht. Darüber sei im folgenden einiges diskutiert. Bei der Hyperfunktion der Schilddrüse ist, wie eingangs erörtert, neben der Steigerung des Gesamtstoffwechsels auch der Vorgang der Eiweisspaltung angeregt. Und die Reaktion des Organismus auf die Fuitterung von Schilddrüsenpräparaten 
scheint sich nach Untersuchungen von Abelin u. Sato ${ }^{7)}$ in einem Phasenverlauf zu äussern. Allenfalls gestalten sich die Verhältnisse hier, so scheint mir, nicht so einfach.

So hat denn $\mathrm{U}$ e $\mathrm{o}^{8}$ ) beim Hyperthyreoidismus eine Abnahme des Albumins und relative Zunahme des Globulins im Blute nachgewiesen; nach Angabe von Morioka ${ }^{13)}$ soll nach Injektionen von Schilddrüsenpräparaten im Blute anfangs Globulin, späterhin Albumin abnehmen. I chika wa u. Sa saki ${ }^{10)}$ fanden beim Hyperthyreoidismus eher eine Vermehrung des Fibrinogens. Yamamoto ${ }^{17}$ an hiesiger Klinik hat bei Kaninchen mit ganz gleicher Methode, wie von mir angewandt wurde, den Hyperthyreoidismus provoziert und in einem beinahe gleichen Versuchsstadium, wie ich die Plasmaphäresis ausführte, an der Leber Bestimmungen des Gesamt-N, Rest-N, Eiweiss-N und des k.o.D. vorgenommen, dabei fand er, dass Eiweiss-N, k.o.D. sowie k.o.D. pro $100 \mathrm{mg} / \mathrm{dl}$ Eiweiss-N abnehmen und Rest-N hingegen erheblich zunimmt.

Demnach wird vorerst der Gedanke nahegelegt, dass es bei der Hyperfunktion der Schilddrüse unter gesteigerter Zersetzung des Eiweisses, also durch gemeinsame Spaltung des Globulin- und Albuminanteils zur Zunahme des Rest-N käme. In demjenigen Fall aber, wo Eiweissstoffe gewaltige Umwälzung erfahren, wie dies bei der Plasmaphäresis der Fall ist, dürfte man eher daran denken, dass die Zersetzung der reifen kleinen Eiweissteilchen mit Leichtigkeit und Übergewicht vonstatten gehe, wogegen unreife, grobere Eiweissteilchen aus Vorratsorganen für das Bluteiweiss angeboten würden; anderseits dürfte auch damit gerechnet werden, dass bei vorgeschrittenem Hyperthyreoidismus nicht allein die Spaltung kleiner Eiweissteilchen vor sich gehe, sondern, dass auch das zu grober Globulinfraktion gehörende Eiweiss direkt, ohne in die osmoaktive Albuminfraktion überzugehen, zum keine Osmoaktivität aufweisenden Rest$\mathrm{N}$ abgespalten würde.

Überigens ist es eine allgemeine Erfahrung, dass beim Ausfall der Schilddrüsenfunktion neben der Herabsetzung des Gesamtstoffwechsels auch der Eiweissstoffwechsel verzögert stattfindet; daraus kann leicht gefolgert werden, dass hierbei auch die Mobilisierung des Eiweisses aus den Vorratsorganen recht langsam erfolgt. Für diesen Umstand spricht allerdings die von mir festgestellte, sehr protrahierte Wiederherstellung des Bluteiweisses. Auch der Grund für die erheblich verspätete Wiederherstellung des k.o.D. ist höchstwahrscheinlich darin zu erblicken, dass die Regulierung der Eiweissteilchen in den Vorratsorganen des Bluteiweisses überaus gestört ist.

17) Yamamoto, wird bald in dieser Zeitschrift erscheinen. 


\section{Schluss.}

1. Wenn am hyperthyreotischen Kaninchen die Plasmaphäresis ausgeführt worden ist, so kann das Eiweiss des Blutes gegenüber der Norm, einigermassen verzögert, doch immerhin wiederhergestellt werden, während die Wiederherstellung des kolloid-osmotischen Drucks hingegen ganz unmöglich erfolgt, wobei der Druck pro $\%$ erheblich herabfällt.

2. Beim athyreotischen Kaninchen, das nachträglich der Plasmaphäresis unterworfen worden ist, findet die Wiederherstellung des Eiweisses des Blutes, des kolloid-osmotischen Drucks sowie des Drucks pro \%o stark verzögert statt. Aber ist diese verzögerte Wiederherstellung des kolloid-osmotischen Drucks und des Drucks pro \% mehr ausgeprägt als die des Bluteiweisses, so dass beide selbst nach 10tägigem Ablauf nach Ausführung der Plasmaphäresis noch nicht vollständige Wiederherstellung erlangen können.

Zum Schluss möchte ich Herm Professor Dr. T. Kato und Herrn Assistant-Professor Dr. F. Na kazawa. a.o. Professor an hiesiger Klinik für ihre freundliche Leitung bei dieser Arbeit aufrichtigsten Dank aussprechen. 